EESTI NSV TEADUSTE AKADEEMIA TOIMETISED 1953. II k., nr. 2 ИЗВЕСТИЯ АКАДЕМИИ НАУК ЭСТОНСКОИ ССР 1953. ТОМ II, 스 2

\title{
ПЛОСКАЯ СТРУЯ ВО ВНЕШНЕМ ПОПЕРЕЧНОМ ПОТОКЕ ВОЗДУХА
}

\author{
ю. В. ИВАНОВ, \\ кандидат технических наук
}

Необходимость определения траекторий турбулентных плоских струй воздуха во внешнем поперечном потоке возникает в связи с расчетом острого дутья, воздушных завес, ғазовых горелок, опылителей, шахтных печей и в других специальных случаях.

Решение этой задачи в общем виде встречает большие математические трудности. Опубликованные аналитические исследования $(1-4,8-11)$ связаны с рядом допущений, ограничений и гипотез, не всегда отражающих физнческую сушность явления. Все эти работы не дают согласуюшихся между собой решений. Более плодотворным путем исследования рассматриваемого случая является путь экспериментальный, с последуюшим сравнением накопленного опытного материала с существующими аналитическими решениями.

В данной статье излагается экспериментальное исследование траекторий изотермических и нензотермических плоских воздушных струй во внешнем поперечном однородном потоке.

При постановке опытов мы исходили из установленного различными исследователями факта незавнсимости закономерностей свободных турбулентных струй от критерия Рейнольдса $(R e)$.

Опыты Д. Н. Ляховского и С. Н. Сыркина $\left({ }^{7}\right)$ с неизотермическими струями показали, что при числе Архимеда $(A r)$ меньше 0,005 можно пренебречь искривлением струй, вытекающих в неподвижное пространство. Ограничив проведение опытов со струями при $\mathrm{Ar}$ меньше 0,005, мы тем самым можем исключить и это влияние.

При отсутствии влияния критернев $R e$ и $A r$ траектории струй, построенные в относительных координатах, следует поставнть в зависимость от следующих определяющих параметров:

1) угла атаки струи $(\alpha)$,

2) коэффициента структуры, струи (a),

3) параметра $\frac{\varrho_{1} v_{1}^{2}}{\varrho_{2} v_{2}^{2}}$.

Безразмерный параметр $\frac{\varrho_{1} v_{1}^{2}}{\varrho_{2} v_{2}^{2}}$ является соотношением количеств движения секундного расхода потока и струи или соотношением их скоростных напоров. Индекс $\alpha 1$ в относится к потоку, а индекс «2» к струе. Әтот параметр был предложен нами $(5,6)$ и оказался полезным при обработке траекторий изотермических и неизотермических круглых струй. 
В этой работе * предполагалось также проверить обобщение траекторий плоских струй по данному параметру.

Коэффнциент $a$ структуры плоских струй, введенный проф. Г. Н. Абрамовичем, дал возможность обобщить струи с различной начальной турбулентностью. Поэтому при изучении струй разной турбулентности коэффициент $a$ войдет в качестве параметра.

Также в качестве самостоятельного параметра должен войти угол атаки струй $\alpha$, так как при переходе от одного угла атаки к другому мы, по существу, переходим к новым условиям перемешнвания. Кроме того, траекторня струн непосредственно зависит от первоначального направления струи в устье.

В качестве характерного линейного размера плоских струй во внешнем поперечном потоке принимаем полуширину сопла в устье $b_{0}$. Уравнение траекторий плоской струи во внешнем поперечном потоке требуется найти в виде:

$$
\frac{y}{b_{0}}=f\left(\frac{x}{b_{0}} ; a ; \frac{\varrho_{1} v_{1}^{2}}{\varrho_{2} v_{2}^{2}} ; a\right) \text {, }
$$

где $x$ и $y$ - расстояния вдоль осей координат от полюса в центре устья сопла до оси струи в произвольном поперечном ее сечении;

$a-$ угол атаки (между струей н потоком), принятый равным нулю, когда поток и струя движутся параллельно и в одном направленни, и равным $180^{\circ}$, когда поток и струя движутся навстречу один другому;

Q1, $v_{1}$ - плотность и скорость потока;

Q2, $v_{2}-$ плотность и скорость на оси струи в устье.

\section{Описание экспериментальной установки и измерений}

На фиг. I показана экспериментальная установка. Основная часть ее состоит из топки, газового воздухоподогревателя, электронагревателей, вентиляторов и набора сопел.

Внешний воздушный поток (с неравномерностью скоростей около $\pm 5 \%$ ) выходил из вертикального канала сечением $480 \times 600$ мм. В этот свободный поток, двигающийся снизу вверх, вдувалась через сопло воздушная струя. По мере своего продвижения под воздействием внешнего потока струя изгибалась. Сопла имели прямоугольную форму и ориентировались большей стороной устья поперек потока. Такие сопла носят названне плоских.

С одной стороны внешний поток ограничивался фронтовой стеной высотой 1250 мм, служившей местом установки сопел. Сопла были съемные и могли ставиться под различными углами атаки к внешнему потоку.

В опытах производились скоростные и температурные замеры вдоль оси струн и в поперечных ее сечениях. По скоростным и температурным замерам определялись дальнобойность струи, изменение скорости и температуры вдоль оси струи и траектория скоростной оси струи.

Под дальнобойностью струи $D_{1}$ понимается относительное расстояние $\frac{x}{b_{0}}$ по нормали от плоскости устья до места струи, в котором проекция осевой скорости на ось $x$ составляет $5 \%$ устьевой скорости.

- При проведенни работы в Центральном научно-исследовательском котло-турбинном ннституте (ЦКТИ) автором были получены ценные советы өт проф. В. С. Жуковского н кандндата технических наук С. С. Кутателадзе. 

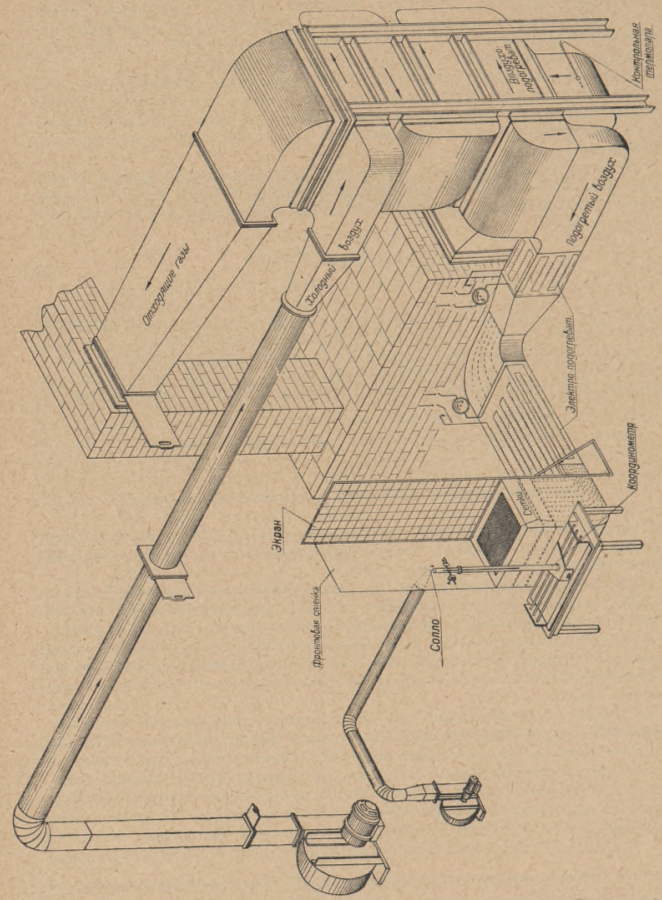

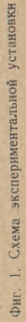


Траектории струй, найденные по замерам, могли сравниваться с траекторнями, определенными по фотографиям окрашенных дымом струй.

Изменение плотности струи в устье производилось регулированием ее температуры при помощи электронагревателей.

Измерения температуры струи пронзводились нихром-константановыми термопарами из проволочек диаметром 0,1 мм.

Скорость струи измерялась изогнутой трубкой с лимбом, имеющим деления для отсчета угла поворота *. Значения скоростей в опытах менялись от 3,5 до $80 \mathrm{~m} /$ сек.

Во время опыта осуществлялся периодический контроль температуры и скорости струи в устье.

Как указывалось выше, струя под влиянием скоростного напора потока изгибалась, поэтому измерения температуры и скорости вдоль ее оси представляли некоторые особенности. Сечения, в которых измерялись скорость и температура, выбирались по перпендикулярному направлению к вектору максимальной скорости струи. В каждой из исследуемых струй выбиралось от пяти до десяти сечений, ориентированных под различными углами $\varphi$ к горизонту.

Изогнутая ось струи, проходящая через точки, где наблюдалось максимальное значение скорости в поперечных сечениях, названа траекторией струи.

Расстояние от устья до сечений выражалось отношением $\frac{L}{b_{0}}$ (длины траектории струи $L$ к полуширине сопла в устье $b_{0}$ ).

Опыты проводились с соплами двух типов, ориентированными под углами к горизонту $\alpha=90^{\circ}$ и $120^{\circ}$, с коэффициентами структуры $a$, равными 0,08 и 0,12 .

Макснмальная относительная ошибка в опытах при измерении температуры составляла $2 \%$, а при измерении скорости $4 \%$.

\section{Результаты опытов}

Со струями во внешнем поперечном потоке было проведено 25 опытов при четырех значениях отношения скоростей струи и потока $\frac{v_{2}}{v_{1}}(5$; $7,1 ; 10 ; 20)$ и при двух значениях отношения температур $\frac{T_{2}}{T_{1}}$ (1 и 2). Прй этом параметр $\frac{\rho_{1} v_{1}^{2}}{\rho_{2} v_{2}^{2}} \quad$ изменялся от 0,0025 до 0,08 , т. е. более чем в 30 раз.

Опыты проводились с тремя соплами прн $\frac{h_{1}}{b_{0}}=10 ; 33$ и 44,5, где $h_{1}$ - бо́льшая сторона сопла в устье. Абсолютные размеры нсследуемых сопел $b_{0}$ составляли 0,$9 ; 2,7$ и 4 мм. Изменение ширины сопел более чем в четыре раза являлось достаточным для того, чтобы подтвердить или опровергнуть возможность обобщения плоских струй в потоке, при отнесении всех линейных размеров к полуширине сопла в устье.

В отличие от затопленных струй, за осевую скорость струи во внешнем однородном потоке принята не абсолютная скорость на оси струи

- Краткое опнсанне нзмерительной трубки дано в работе автора ${ }^{(6)}$. 
$v_{m}$, а разность скоростей $v_{m}-v_{1}$, где $v_{1}-$ скорость потока. 3а осевую скорость струи в устье также принята разность скоростей $v_{0}-v_{1}$, где $v_{0}-$ скорость на оси струи в устье.
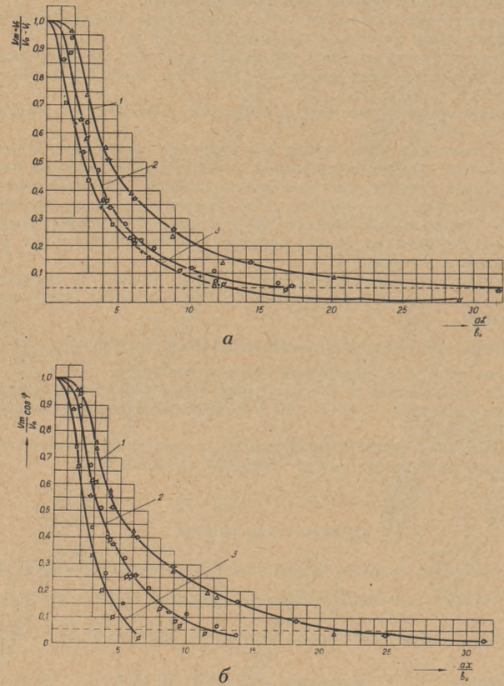

Фнг. 2. Изменення разностей и проекций осевых скоростей плоскнх струй во внешнем потоке

a- разности осевых скоростей при $\frac{T_{2}}{T_{1}}=1$ и $a=90^{\circ}$;

$$
1-\frac{v_{2}}{v_{1}}=20 ; \quad 2 \quad \frac{v_{2}}{v_{1}}=10 ; \quad 3-\frac{v_{2}}{v_{1}}=5
$$

6 - проекции осевых скоростей при тех же условиях.

Изменение безразмерной осевой скорости $\frac{v_{m}-v_{1}}{v_{0}-v_{1}}$ в зависимости от безразмерного расстояния $\frac{a L}{b_{0}}$ показано на фиг. 2, $a$. Сравнивая кривые при различных отношениях скоростей, можно видеть, что, чем меньше 
отношение $\frac{v_{2}}{v_{1}}$, тем быстрее падает безразмерная осевая скорость струи. Из графика видно, что точки, полученные из опытов со струямн, имеющими различные значения коэффициентов структуры $a$, обобщаются в единые крнвые.
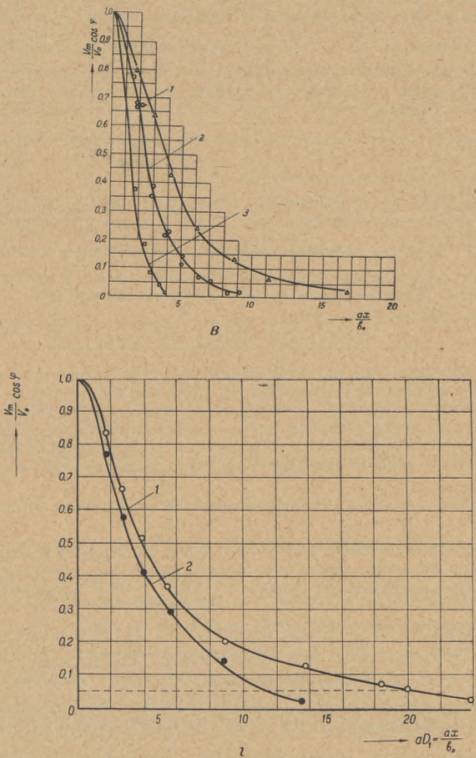

Фит. 2. Изменения разностей и проекций осевых скоростей плоскнх струй во внешнем потоке

в- проекцин осевых скоростей прн $\frac{T_{2}}{T_{1}}=2$ и $a=90^{\circ} ; t-\frac{v_{y}}{v_{1}}=20$;

$$
2-\frac{v_{2}}{v_{1}}=10: \quad 3-\frac{v_{2}}{v_{1}}=5
$$

2 - проекцин осевых скоростей прн $\frac{T_{2}}{T_{1}}=1$ и $a=120^{\circ} ; \quad 1-\frac{v_{9}}{v_{1}}=20 ; \quad 2-\frac{v_{9}}{v_{1}}=10$ 
Для того чтобы найти количественные зависимости затухания плоских струй, развивающихся во внешнем однородном потоке, используем введенное выше понятие о дальнобойности струй $D_{1}$. С этой целью на фиг. 2,6 и 2,8 построены проекции осевых скоростей на горнзонтальную ось $\frac{v_{m}}{v_{0}} \cdot \cos \varphi$ В зависимости от безразмерной величины $\frac{a x}{b_{0}}$.

Кривые на фиг. 2, б относятся к холодным струям, а на фиг. 2, в к горячим струям при $\frac{T_{2}}{T_{1}}=2$. Если сделать сравнение проекций скоростей вдоль оси горячей н-холодной плоских струй при одинаковых отношениях скоростей, то видно, что горячие струи затухают быстрее. Из приведенных графиков фиг, 2 можно сделать вывод, что струя в потоке теряет скорость на оси тем быстрее, чем меньше отношение скоростей $\frac{v_{2}}{v_{1}}$ и чем больше отношение температур $\frac{T_{2}}{T_{1}}$.

Произведения из коэффициента структуры струй $a$ на дальнобойность струй $D_{1}$ определяются из фиг. 2,6 и 2,8 как относительные расстояния $\frac{a x}{b_{v}}$ в местах пересечения кривых с пунктирными линиями, проведенными на уровне проекции осевой скорости на ось $x$, составляющей $5 \%$ устьевой скорости. Значения $a D_{1}$ из шести кривых фиг. 2, 6 и 2,8 сведены в таблицу 1.

\begin{tabular}{c|c|c|c|c|c|c|}
\hline & \multicolumn{3}{|c|}{$T_{2}=1$} & \multicolumn{3}{|c|}{$\frac{T_{2}}{T_{1}=2}$} \\
\hline & \multicolumn{3}{|c|}{$T_{1}$} \\
\hline$v_{1}$ & 20 & 10 & 5 & 20 & 10 & 5 \\
$a D_{1}$ & 24 & 12 & 6 & 14 & 7,7 & 3,5
\end{tabular}

таблица 1

Табличные значения $a D_{1}$ представлены в зависимости от параметра $\rho_{2} v_{2}^{2}$ в логарифмическом масштабе на фиг. 3. Экспериментальные точки струй, выходящих из сопел с $\frac{h_{1}}{b_{0}}=33$ и 44,5, укладываются на одну прямую. Значения $a D_{1}$ струй, выходящих из сопел $\mathrm{c} \frac{h_{1}}{b_{0}}=10$, получились меньше на $\sim 20 \%$. Это расхождение объясняется тем, что струи, выходящие из сопел с отношением сторон $\frac{h_{1}}{b_{0}}=10$, затухают быстрее, чем струи, выходящие из более вытянутых сопел. Значения дальнобойностей струй, выходящих из этих сопел, приведены потому, что в нашем распоряжении не было других опытов при $\frac{T_{2}}{T_{1}}=2$. В то же время опыты с горячими струями характерны тем, что они в основном подчиняются той же зависимости от параметра $\frac{\varrho_{2} v_{2}^{2}}{\varrho_{1} v_{1}^{2}}$, как и холодные струн. Учитывая, однако, что нас интересуют дальнобойности плоских вытянутых струй, прямая на фиг. 3 проведена по точкам, относящимся к струям, выходящим из сопел с $\frac{h_{1}}{b_{0}}=33$ и 44,5 . 


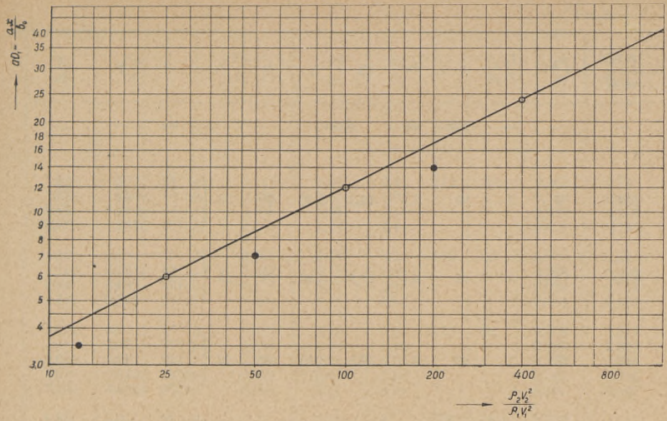

Фиг. 3. Дальнобойность плоских струй при $\alpha=90^{\circ}$ в занисимости от соотношения скоростных напоров струн и потока

Представленная в логарифмической сетке на фиг. 3 зависимость показывает, что значения $a D_{1}$ струй укладываются на прямую с показателем степени 0,5 . Так как опыты проводились со струями, имеющнми широкий диапазон изменения значения коэффициента структуры, то можно рекомендовать формулу для определения дальнобойности плоских вытянутых струй с различными коэффнциентами структуры в виде

$$
a D_{1}=k_{2} \frac{v_{2}}{v_{1}} \sqrt{\frac{\omega_{2}}{\rho_{1}}},
$$

где коэффициент пропорщиональности $k_{2}=1,2$ при угле атаки $\alpha=90^{\circ}$.

Коэффициент пропорциональности для струй, ориентированных под углом $a=120^{\circ}$, можно определнть из кривых фит. 2, г. Так, при $\frac{v_{y}}{v_{1}}=20$ получается $a D_{1}=22$, при $\frac{v_{y}}{v_{1}}=10$ имеем $a D_{1}=11$. Подставляя эги значения в формулу (2), нолучим при угле атаки $\alpha=120^{\circ}$ значение $\mathrm{k}_{2}=1,1$. В опытах с круглыми соплами $\left({ }^{5}\right)$ максимальной дальнобойностью обладали струи с углом атаки $\alpha=90^{\circ}$, так что полученное в этих опытах уменышение дальнобойности при $\alpha=120^{\circ}$ находится в полном соответствии с результатами-вышеуказанной работы.

\section{Траектории плоских струй}

Основной задачей данного исследования, как уже было сказано выше, являлось экспериментальное определение уравнения траекторий плоских струй, развивающихся во внешнем поперечном потоке.

Исходя из того, что струи при $\frac{h_{1}}{b_{0}}=10$ затухают быстрее вытянутых 


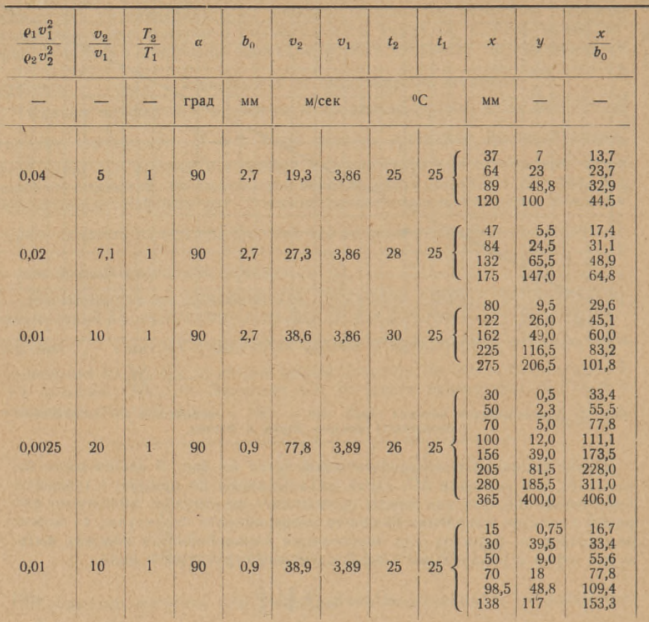

плоских струй, мы определяем уравнение траекторий струй из опытов с соплами $\frac{h_{1}}{b_{0}}=33$ и 44,5 . Данные этих опытов приведены в таблице 2.

Обработка траекторий струй этих опытов сделана согласно предложению Г. Н. Абрамовича $\left({ }^{1,2}\right)$ в координатах

$$
\frac{a x}{b_{0}}, \frac{a y}{b_{s}} .
$$

Полученные кривые показывают, что траектории струй с различной начальной турбулентностью введением коэффициента структуры $a$ обобщаются в единые крнвые. Так, например, кривая 3 на фиг. 4 образована точками опытов со струями $a=0,08$ и 0,12 при $\frac{h_{1}}{b_{n}}=33$ и 44,5.

Уравнение траектории струи при угле атаки $\alpha=90^{\circ}$ будем искать в виде степенной зависимости:

$$
\frac{a y}{b_{0}}=c\left(\frac{\varrho_{1} v_{1}^{2}}{\varrho_{2} v_{2}^{2}}\right)^{m}\left(\frac{a x}{b_{0}}\right)^{n} .
$$

Для нахождения постоянных $c, m$ и $n$ в уравненим (3) на фиг. 5 перестроены в логарифмической сетке графические зависимости фиг. 4. 
Taблuца 2

\begin{tabular}{|c|c|c|c|c|c|c|c|c|c|}
\hline$\frac{y}{b_{0}}$ & $\frac{v_{m}}{v_{s}}$ & $\varphi$ & $\frac{v_{m}-v_{1}}{v_{0}-v_{1}}$ & $\frac{a x}{b_{0}}$ & $\frac{a y}{b_{0}}$ & $a$ & $\frac{h_{1}}{b_{0}}$ & $\cos \varphi$ & $\frac{v_{m}}{v_{0}} \cos \varphi$ \\
\hline- & - & град & - & - & - & - & - & - & - \\
\hline $\begin{array}{c}2,59 \\
8,51 \\
18,1 \\
37,0\end{array}$ & $\begin{array}{l}0,771 \\
0,546 \\
0,429 \\
0,326\end{array}$ & $\begin{array}{l}16 \\
36 \\
52 \\
63\end{array}$ & $\begin{array}{l}0,714 \\
0,434 \\
0,286 \\
0,156\end{array}$ & $\begin{array}{l}1,64 \\
2,85 \\
3,95 \\
5,34\end{array}$ & $\begin{array}{l}0,31 \\
1,02 \\
2,17 \\
4,44\end{array}$ & 0,12 & 33 & $\begin{array}{l}0,961 \\
0,809 \\
0,616 \\
0,454\end{array}$ & $\begin{array}{l}0,74 \\
0,44 \\
0,264 \\
0,148\end{array}$ \\
\hline $\begin{array}{c}2,04 \\
9,08 \\
24,2 \\
54,5\end{array}$ & $\begin{array}{l}0,69 \\
0,436 \\
0,294 \\
0,22\end{array}$ & $\begin{array}{l}14 \\
25 \\
52 \\
63\end{array}$ & $\begin{array}{l}0,637 \\
0,342 \\
0,177 \\
0,09\end{array}$ & $\begin{array}{l}2,09 \\
3,73 \\
5,87 \\
7,78\end{array}$ & $\begin{array}{l}0,24 \\
1,09 \\
2,91 \\
6,54\end{array}$ & 0,12 & 33 & $\begin{array}{l}0,970 \\
0,906 \\
0,616 \\
0,454\end{array}$ & $\begin{array}{l}0,67 \\
0,395 \\
0,181 \\
0,099\end{array}$ \\
\hline $\begin{array}{c}3,52 \\
9,63 \\
18,34 \\
43,1 \\
76,5\end{array}$ & $\begin{array}{l}0,525 \\
0,358 \\
0,271 \\
0,095 \\
0,159\end{array}$ & $\begin{array}{l}12 \\
26 \\
40 \\
57 \\
67\end{array}$ & $\begin{array}{l}0,472 \\
0,288 \\
0,190 \\
0,106 \\
0,065\end{array}$ & $\begin{array}{c}3,55 \\
5,41 \\
7,26 \\
10,0 \\
12,2\end{array}$ & $\begin{array}{l}0,42 \\
1,16 \\
2,18 \\
5,18 \\
9,18\end{array}$ & 0,12 & 33 & $\begin{array}{l}0,978 \\
0,899 \\
0,766 \\
0,545 \\
0,391\end{array}$ & $\begin{array}{l}0,512 \\
0,322 \\
0,207 \\
0,106 \\
0,062\end{array}$ \\
\hline $\begin{array}{c}\overline{2,5} \\
5,55 \\
13,32 \\
43,4 \\
90,6 \\
206,0 \\
445,0\end{array}$ & $\begin{array}{l}\overline{0}, 516 \\
0,408 \\
0,302 \\
0,189 \\
0,131 \\
0,088 \\
0,068\end{array}$ & $\begin{array}{r}2 \\
7 \\
9 \\
14 \\
31 \\
50 \\
66 \\
82\end{array}$ & $\begin{array}{l}0,505 \\
0,376 \\
0,265 \\
0,146 \\
0,087 \\
0,040 \\
0,019\end{array}$ & $\begin{array}{r}2,67 \\
4,44 \\
6,23 \\
8,89 \\
13,87 \\
18,23 \\
24,9 \\
32,4\end{array}$ & $\begin{array}{l}0 \\
0,20 \\
0,444 \\
1,065 \\
3,47 \\
7,25 \\
16,48 \\
35,5\end{array}$ & 0,08 & 44,5 & $\begin{array}{l}0,999 \\
0,993 \\
0,998 \\
0,970 \\
0,857 \\
0,643 \\
0,407 \\
0,139\end{array}$ & $\begin{array}{l}\overline{0,512} \\
0,404 \\
0,293 \\
0,162 \\
0,084 \\
0,036 \\
0,009\end{array}$ \\
\hline $\begin{array}{r}0,83 \\
3,61 \\
10,0 \\
20,0 \\
54,2 \\
130,0\end{array}$ & $\begin{array}{l}0,89 \\
0,584 \\
0,414 \\
0,3112 \\
0,206 \\
0,147\end{array}$ & $\begin{array}{l}1 \\
10 \\
22 \\
32 \\
55 \\
\end{array}$ & $\begin{array}{l}0,868 \\
0,532 \\
0,345 \\
0,223 \\
0,117 \\
0,052\end{array}$ & $\begin{array}{r}1,33 \\
2,67 \\
4,45 \\
6,23 \\
8,75 \\
12,25\end{array}$ & $\begin{array}{c}0,067 \\
0,289 \\
0,80 \\
1,6 \\
4,33 \\
10,4\end{array}$ & 0,08 &.$^{44,5}$ & $\begin{array}{l}0,999 \\
0,985 \\
0,927 \\
0,848 \\
0,574 \\
-\end{array}$ & $\begin{array}{l}0,889 \\
0,575 \\
0,384 \\
0,256 \\
0,118 \\
-\end{array}$ \\
\hline
\end{tabular}

На фиг. 5 видно, что опытные точки, характеризующиеся одннаковыми значениями параметра $\frac{\rho_{1} v_{1}^{2}}{\rho_{2} v_{2}^{2}}$, располагаются на одной прямой, и прямые с разными значениями $\frac{\vartheta_{1} v_{1}^{2}}{\rho_{2} v_{2}^{2}}$ являются параллельными между собою. По наклону этих прямых устанавливаем, что показатель степени $n=2,5$. Дальнейшей обработкой этих графиков на фнг. 6 получаем $c=1,9$ и $m=1$.

Итак, уравнение траекторий плоских струй, при угле атаки $\alpha=90^{\circ}$, развивающихся во внешнем поперечном потоке, будет иметь вид:

$$
\frac{a y}{b_{0}}=1,9 \frac{\rho_{1} v_{1}^{2}}{\rho_{2} v_{2}^{2}}\left(\frac{a x}{b_{0}}\right)^{2,5} .
$$

На фиг. 7 проведено сравнение траекторий струй, полученных по уравнению (4), с экспериментальными точками всех опытов.

Рассматривая графики траекторий струй (фиг. 7), можно сделать следующие выводы: 
1. Плоские струи, выходящие из сопел с $\frac{h_{1}}{b_{0}}=10$, подчиняются тому же уравнению (4), но, как правило, они лежат несколько выше, чем точки, полученные в опытах с соплами $\frac{h_{1}}{b_{0}}=33$ и 44,5. Объясняется это, как уже было сказано, бо́льшим. затуханием струй, выходящих из сопел с отношением сторон $\frac{h_{1}}{b_{0}}<30$.

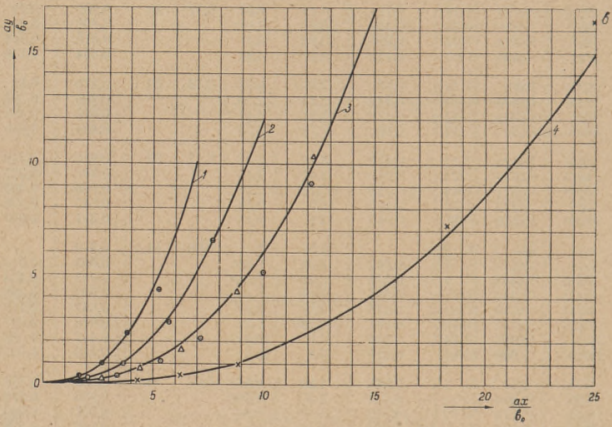

Фит. 4. Траекторнн плоских струй при $\alpha=90^{\circ}\left(\frac{h_{1}}{b_{0}}=33\right.$ и 44,5$)$

2. Плоские струи вытянутой формы (при $\frac{h_{1}}{b_{0}} \geqslant 30$ ), развивающиеся в однородном внешнем потоке, можно также, как плоские свободные струи (т. е. струи, вытекающие в неподвижное пространство тех же фнзических свойств), обобщать по $\mathrm{b}_{0}$.

3. Общим для каждой кривой является параметр $\frac{\rho_{1} v_{1}^{2}}{\varrho_{2} v_{2}^{2}}$. Действительно, большинство кривых объединяет экспериментальные точки различных опытов с холодными и горячими струями, проведенных при различных отношениях скоростей. Важно только абсолютное значение этого параметра, несмотря на то, что он составлен из различных комбинаций входящих в него величин. Таким образом, целесообразность введения этого параметра подтвердилась опытами.

4. Полученное уравнение (4) можно считать проверенным в интервале значений параметра $\frac{\varrho_{1} v_{1}^{2}}{\varrho_{2} v_{2}^{2}}$ oт 0,0025 до 0,08 и значений $a$ от 0,08 до 0,12 .

При пользовании уравнением (4) для расчета траекторий струй острого дутья $\mathbf{3}$ топке предлагается ограничить применение его до рас- 


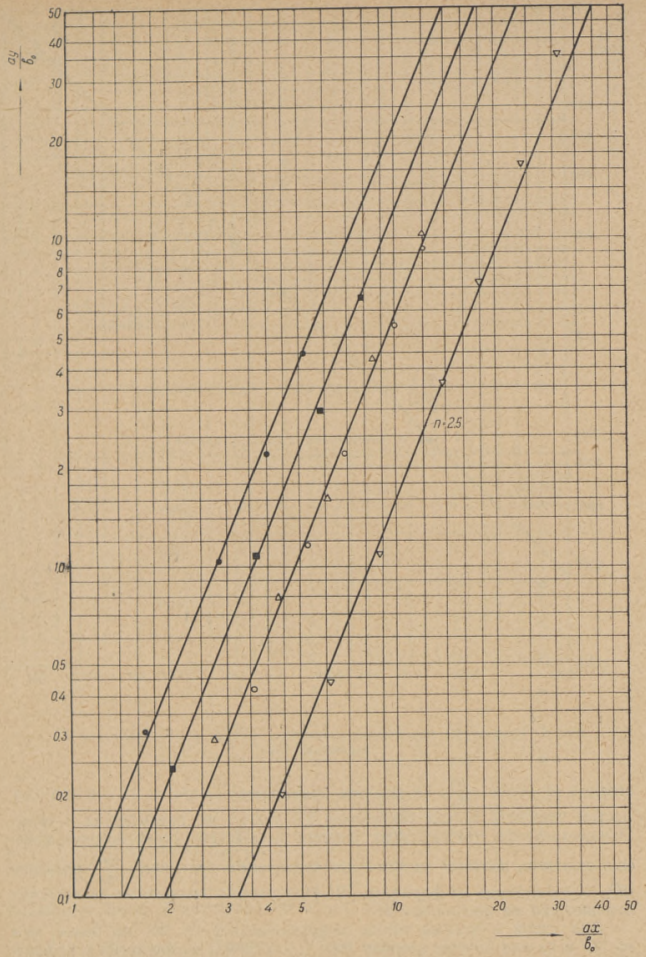

Фиг, 5, График для определения показателя степенн $n$ в уравнении (3) 


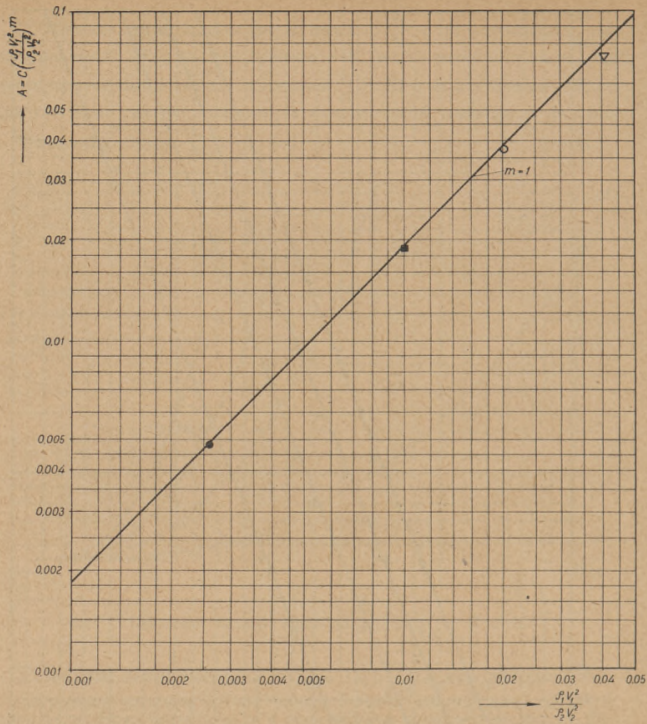

Фиг, 6. Графнк лия определения показателя стелени $m \|$ козфициента $c$ в уравненин (3)

стояния от устья $\frac{a x}{b_{0}}$, на котором превышение скорости на оси над скоростью внешнего потока составляет $5 \%$ такого же превышения в устье. Предполагается, что при этом условии воздействие струй острого дутья еще ощутимо, поэтому практически предельным значением $\frac{a x}{b_{0}}$ является $a D_{1}$, определяемое из формулы (2). При расчете воздушных завес таких ограничений не предлагается.

Для струй с углами атаки, отличающимися от $90^{\circ}$ не более, чем на $30^{\circ}$ в ту или другую сторону $\left(60^{\circ} \leqslant \alpha \leqslant 120^{\circ}\right)$, по аналогии с работой ${ }^{(5)}$ можно рекомендовать уравнение траекторий струй в виде:

$$
\frac{a y}{b_{0}}=1,9 \frac{e_{1} v_{1}^{2}}{\varrho_{0} v_{2}^{2}}\left(\frac{a x}{b_{0}}\right)^{2,5}-\frac{a x}{b_{0}} \operatorname{tg}\left(a-90^{\circ}\right) .
$$




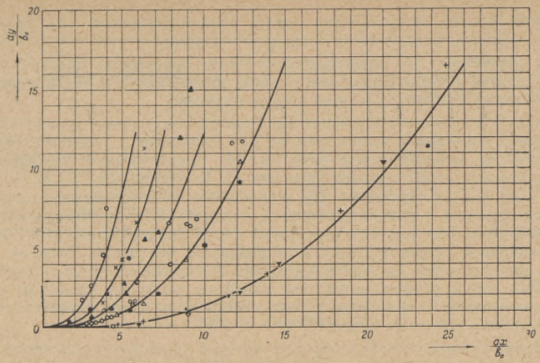

Фиг. 7. Граекторин плоских струй при $\alpha=90^{\circ}\left(\frac{h_{1}}{b_{9}}=10 ; 33\right.$ и 44,5$)$

Таблица условных обозначений к фиг. 7

\begin{tabular}{|c|c|c|c|c|c|c|}
\hline$\frac{\text { 로을 }}{\text { кривых }}$ & $\frac{\varrho_{1} v_{1}^{2}}{\varrho_{2} v_{2}^{2}}$ & $\frac{v_{2}}{v_{1}}$ & $\frac{T_{2}}{T_{1}}$ & $a$ & $\frac{h_{1}}{b_{1}}$ & $\begin{array}{c}\text { Условные } \\
\text { обозна- } \\
\text { чения }\end{array}$ \\
\hline 1 & 0,08 & 5 & 2 & 0,12 & 10 & 0 \\
\hline \multirow{2}{*}{2} & \multirow{2}{*}{0,04} & 5 & 1 & 0,12 & 33 & $\theta$ \\
\hline & & 5 & 1 & 0,12 & 10 & $x$ \\
\hline \multirow{2}{*}{3} & \multirow{2}{*}{$0, \overline{02}$} & 7,1 & 1 & 0,12 & 33 & 0 \\
\hline & & 10 & 2 & 0,12 & 10 & $\Delta$ \\
\hline \multirow{3}{*}{4} & \multirow{3}{*}{0,01} & 10 & 1 & 0,08 & 44,5 & $\Delta$ \\
\hline & & 10 & 1 & 0,12 & 33,0 & 0 \\
\hline & & 10 & 1 & 0,12 & 10,0 & 0 \\
\hline \multirow[t]{2}{*}{5} & \multirow[t]{2}{*}{0,0025} & 20 & 1 & 0,08 & 44,5 & + \\
\hline & & 20 & 1 & 0,12 & 10,0 & $\nabla$ \\
\hline
\end{tabular}

Уравнение верно в интервале $60^{\circ} \leqslant \alpha \leqslant 120^{\circ}$ при $0 \leqslant \frac{a x}{b_{0}} \leqslant a D_{1}$ и $0,0025 \leqslant \frac{e_{1} v_{1}^{2}}{\rho_{2} v_{2}^{2}} \leqslant 0,08$.

Второй член правой части уравнения (5) добавлен в качестве величины, учитывающей влияние угла атаки. Например, при отсутствни внешнего потока первый член правой части уравнения (5) равен нулю 
(так как $v_{1}=0$ ) и траектории струй, ориентированные пол разными углами атаки $a$, описываются вторым его членом.

По уравнению (5) можно рассчитывать траектории плоских струй острого дутья в топках, воздушных завес, применяемых в вентиляционноотопительной технике, и в ряде других случаев.

Приведем несколько примеров применения полученного уравнения для численных расчетов и используем их для сравнения с расчетами по существуюшим аналитическим работам. Из существующих современных аналитических работ возьмем решения И. К. Наймарка $\left({ }^{8}\right)$, И. А. Шепелева $\left({ }^{9}\right)$ и С Е. Бутакова $\left({ }^{4}\right)$, основанные на совершенио различных методах подхода к рассматриваемому явлению. И. К. Наймарк предложил формулы для расчета траекторий струй острого дутья при заданной начальной скорости струи и направлении оси сопла. Выводы формулы основываются на закономерностях затопленных струй, которые в условиях топки искривляются под влняннем разности удельных весов вдуваемого воздуха и горячих газов, а также благодаря воздействию на струю потока топочных газов.

Второй аналитической работой, с которой приводится сравнение, является аналитическое исследование И. А. Шепелева $\left({ }^{9}\right)$, опубликованное в 1947 г. В этой работе предлагается метод расчета воздушных завес. Функция тока завесы (как результат взаимодействия струи с набегающим потоком) может быть определена простым сложением функций тока струи и набегающего потока. Этот прнем годится для потенциальных потоков и вряд ли можно предполагать прнменимость его для реальных воздушных потоков.

Третьей работой, с которой приводится сравненне, является аналитическое исследование С. Е. Бутакова $\left({ }^{4}\right)$. Последний считает, что существующне методы определения траектории воздушной завесы основаны на чисто кинематических соображениях, и указывает совершенно правильно, что такой подход не является единственно возможным.

Движение воздуха в проемах вызывается в основном ветром, который никогда не врывается непосредственно в проемы зданий. Он создает y ограждений зданий зоны повышенных и пониженных давлений, отличающихся от давления в помещении; разности давлений создают движение воздуха в проемах или выгибают осн перекрывающих их завес. Исходя из сказанного, С. Е. Бутаков предлагает метод расчета воздушных завес, в котором разность давлений рассматривается как основной фактор, обуславливающий выгиб завес. Однако, прежде чем рекомендовать этот способ для практического применения, автор считает необходимым его уточнить на основе экспериментов.

Сравнение экспернментальных данных проведенного исследования с вышеуказанными тремя аналитическими работами сделано на примерах, прнведенных в табл. 3. Порядок расчетов по уравнению (5) для двух примеров приведен в табл. 4. Там же, в правой части таблицы, даны для сравнения координаты оси завесы $x$ н $y$, взятые из аналитических работ $\left({ }^{4,8}\right)$.

Графическое сравнение траекторий струй, построенных по уравнению (5), с траекториями, определенными по аналитическим работам для приведенных в таблице 4 условий, сделано на фиг. $8, a ; 8,6$ и $8, \boldsymbol{s}$.

На фиг. 8, a построены две траектории оси струи острого дутья, сплошная кривая по уравнению (5) и пунктирная кривая по уравнению И. К. Наймарка. Из сравнения кривых видно, что по уравнению И. К. Наймарка струя более дальнобойна. 


\section{Данные}

Ширина щели, $b_{0}$

Угол атаки струи, $a$

Скорость струн в устье, $v_{2}$

Скорость внешнего потока, $v_{1}$

Температура струи, $T_{2}$

Температура внешнего потока, $T_{1}$

Коэффишиент структуры струи, $a$

И. К. Наймарка С. Е. Бутакова

Един.

แ3м.

изм. И. К. Наймарка

M
rрaд.
M/сек
м/сек
абс
абс

0,008
120
50
3,3
550
1800
0,1

0,05125

120

20

4,04

293

293

0,2

Таблища 4

Прнмеры расчетов траекторнй струй острого дутья и воздушных завес по уравнению (5)

$$
\frac{a y}{b_{0}}=1,9 \frac{\varrho_{1} v_{1}^{2}}{\varrho_{2} v_{2}^{2}}\left(\frac{a x}{b_{0}}\right)^{2.5}-\frac{a x}{b_{0}} \operatorname{tg}\left(a-90^{\circ}\right) .
$$

Обозначим $1,9 \frac{\varrho_{1} v_{1}^{2}}{\rho_{2} v_{2}^{2}}\left(\frac{a x}{b_{0}}\right)^{2,5}=A$; тогда при $\alpha=120^{\circ}$

$$
\frac{a y}{b_{0}}=A-0,577 \frac{a x}{b_{11}} .
$$

\begin{tabular}{|c|c|c|c|c|c|c|c|c|c|}
\hline$\frac{a x}{b_{0}}$ & $\left(\frac{a x}{b_{0}}\right)^{2,5}$ & $1,9 \frac{\rho_{1} v_{1}^{2}}{Q_{2} v_{2}^{2}}$ & $A$ & $0,577 \frac{a x}{b_{0}}$ & $\frac{a y}{b_{0}}$ & $x$ & y & $x$ & y \\
\hline - & - & - & - & $=$ & - & M & M & M & M \\
\hline & & & & & & & & \multicolumn{2}{|c|}{$\begin{array}{l}\text { По уравнению } \\
\text { И. К. Нанямарка }\end{array}$} \\
\hline $\begin{array}{c}2 \\
4 \\
6 \\
8 \\
10 \\
12,5 \\
25,0 \\
37,5 \\
50,0 \\
75,0\end{array}$ & $\begin{array}{c}5,65 \\
32,0 \\
88,5 \\
181 \\
316 \\
552 \\
3125 \\
8600 \\
17625 \\
48500\end{array}$ & 0,00255 & $\begin{array}{c}0,0144 \\
0,082 \\
0,226 \\
0,463 \\
0,81 \\
1,41 \\
7,97 \\
22,0 \\
45,2 \\
124,0\end{array}$ & $\begin{array}{r}1,15 \\
2,31 \\
3,46 \\
4,62 \\
5,77 \\
7,20 \\
14,4 \\
21,6 \\
28,9 \\
43,3\end{array}$ & $\begin{array}{l}-1,14 \\
-2,30 \\
-3,23 \\
-4,16 \\
-4,96 \\
-5,79 \\
-6,43 \\
10,4 \\
16,3 \\
80,7\end{array}$ & $\begin{array}{l}0,16 \\
0,32 \\
0,48 \\
0,64 \\
0,8 \\
1,0 \\
2,0 \\
3,0 \\
4,0 \\
6,0\end{array}$ & $\begin{array}{c}-0,091 \\
-0,184 \\
-0,259 \\
-0,333 \\
-0,397 \\
-0,463 \\
-0,515 \\
0,0032 \\
1,31 \\
6,47\end{array}$ & $\begin{array}{l}1,0 \\
2,0 \\
3,0 \\
4,0 \\
6,0\end{array}$ & $\begin{array}{l} \\
-0,355 \\
-0,619 \\
-0,696 \\
-0,692 \\
-0,466\end{array}$ \\
\hline & & & & & & & & \multicolumn{2}{|c|}{$\begin{array}{l}\text { По уравнению } \\
\text { С. Е. Бутакова }\end{array}$} \\
\hline $\begin{array}{c}1 \\
2 \\
4 \\
5 \\
6 \\
7,8 \\
11,7 \\
15,6\end{array}$ & $\begin{array}{c}1,0 \\
5,65 \\
32,0 \\
55,0 \\
88,5 \\
170 \\
465 \\
960 \\
\end{array}$ & 0,078 & $\begin{array}{c}0,078 \\
0,44 \\
2,5 \\
4,29 \\
6,9 \\
13,3 \\
36,3 \\
75,0 \\
\end{array}$ & $\begin{array}{l}0,577 \\
1,15 \\
2,31 \\
2,88 \\
3,46 \\
4,5 \\
6,75 \\
9,00 \\
-\end{array}$ & $\begin{array}{c}-0,50 \\
-0,714 \\
0,19 \\
1,41 \\
3,44 \\
8,8 \\
29,6 \\
66,0 \\
-\end{array}$ & $\begin{array}{l}0,256 \\
0,512 \\
1,02 \\
1,28 \\
1,53 \\
2,00 \\
3,00 \\
4,00 \\
-\end{array}$ & $\begin{array}{c}-0,128 \\
-0,183 \\
0,049 \\
0,36 \\
0,875 \\
2,25 \\
7,50 \\
16,9\end{array}$ & $\begin{array}{l}2,00 \\
3,00 \\
4,00 \\
5,0\end{array}$ & $\begin{array}{c}-0,655 \\
-0,70 \\
-0,47 \\
0\end{array}$ \\
\hline
\end{tabular}


Сравнение экспериментальных данных с аналитической работой И. А. Шепелева приведено на фиг. 8, б. Кривые построены для двух случаев, $\alpha=90^{\circ}$ и $120^{\circ}$. Как видно из графика, траектория струи, построенная по данным аналитической работы И. А. Шепелева (пунктирная кривая), проходит ближе к фронтовой стене, чем экспернментальная кривая.
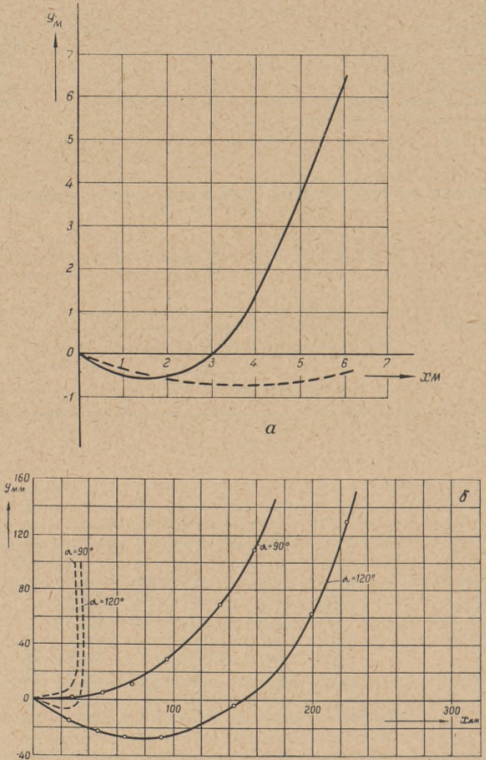

6

Фиг. 8. Сопоставление траекторий плоских струй, построенных по уравненню (5) с траекториямн определенными аналитическим путем

$$
\begin{aligned}
& a — \text { по уравнению (5) } \\
& \text { ㄷ.. по теории И., К. Наймарка } \\
& \ldots \text { по уравненню (5) Но теорни И. А. Шелелева }
\end{aligned}
$$




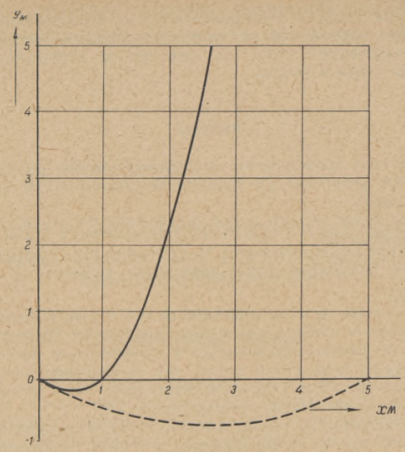

$\boldsymbol{B}$

Фиг. 8. Сопоставление траекторнй плоскнх струй, построенных по уравнению (5) с траекториями определенными аналитическим путем

$$
\begin{aligned}
& \text { в - по уравненио (5) } \\
& \text {... по теорни C. Е. Бутакова }
\end{aligned}
$$

Траектория струи по уравнению С. Е. Бутакова нанесена на фиг. $8, \boldsymbol{\theta}$ пунктирной линией, а по уравнению (5) - сплошной. Как видно из графика, расхождение кривых столь же значительно, как и при сравнении с двумя вышеупомянутыми аналитическими работами.

Из приведенного сравнения видно, что траектория плюской струи, подсчитанная по уравнению (5), занимает промежуточное положение между траекториями, построенными по работе И. А. Шепелева, с одной стороны, и И. К. Наймарка и С. Е. Бутакова - с другой. Из предложенных аналитических методов определения траектории струи в потоке предпочтение следует отдать методу С. Е. Бутакова.

Результаты проведенного экспериментального исследования дают основание предполагать, что траектория струи изгибается под воздействием усилия, которое оказывает скоростной напор набегающего потока $\left(\varrho v^{2}\right)$. Расхождение экспериментальной траектории с расчетной по предложенному С. Е. Бутаковым методу можно объяснить недоучетом некоторых факторов при аналитическом решении. Так, например, затухание струи в потоке взято по закону свободной струн, а по нашим экспериментальным данным видно, что струя в потоке затухает значительно быстрее, чем при затопленном истечении. Затем подсос воздуха с обеих сторон завесы принят одинаковым, в то время как изогнутая струя с внешней и с внутренней стороны взанмодействует с потоком различно. Если этот метод уточнить на основании эксперимента, как и предполагал автор, то им, возможно, удастся пользоваться при практических расчетах. 
На основании данного экспериментального исследования остальные методы решения, основанные на кинематических соображениях, можно считать неоправдавшими себя, так как они не отражают механизма взаимодействия струи и потока.

\section{Выводы}

1. В результате проведенного экспериментального исследования получено расчетное уравнение $\left(^{5}\right)$ траекторий плоских струй острого дутья и воздушных завес.

При расчете струй острого дутья дальнобойность струй следует определять по формуле (2).

При расчете воздушных завес предельное значение $\frac{a x}{b_{0}}$ зависнт. от условий задачи.

2. Падение скорости и температуры по оси струй, развивающихся во внешнем поперечном однородном потоке, происходит интенсивнее, чем у тех же струй, но распространяющихся в неподвижном пространстве.

3. Чем больше угол атаки струи, тем интенсивнее падает ее скорость и температура. При установке воздушных завес нельзя рекомендовать значения углов атаки, превышающие $120^{\circ}$.

4. Олыты подтвердили предположения Г. Н. Абрамовича о возможности обобщения струй, развивающихея в потоке, отнесением всех линейных размеров струи к полуширине струи в устье с учетом коэффициента их структуры.

5. Данные проведенной работы опровергают расчеты траекторнй струй в потоке, основанные на различных методах сложения скоростей струн со скоростью потока.

6. Приведенные примеры расчета показывают удобство и простоту применения уравнения траекторий к практическим случаям.

Ннститут әнергетики

Академии наук Эстонской ССР
Поступнла в редакцию 10 VI 1953

\section{ЛИТЕРАТУРА}

1. Г. Н. Абрамовн ч, Труды ЦАГИ, вып. 293, 1936.

2. Г. Н. Абрамович, Турбулентные свободные струи жидкостей и газов, 1948 , cтp. 200.

3. В. В. Батурнн и И. А. Шелелев, Воздушные завесы, журнал «Отопление н вентиляшия, № $5,1936$.

4. С. Е. Бутаков, Аэродннамика систем промышленной вентиляции, Профиздат. M., 1949, cтp. $162-172$.

5. Ю. В. Ив анов, У равнення траекторнй струй острого дутья, КТС, № 8, 1952.

6. Ю. В. Иванов, Исследованне траекторнй холодных и горячнх струй, распространяюшихся в неограннченном однородном потоке, Диссертащия, 1951.

7. Д. Н. Ляховскнй н С. Н. Сыркин, Аэродинамика факела, вытекающего в среду другой плотности, ЖТФ, № 9, 1939.

8. И. К. Н а йм ар К, К вопросу о расчете острого дутья, СКТС, № 7, 1939.

9. И. А. Шепе лев, Воздушные завесы, Изд. НИИЛ, 1947.

10. И. А. Ш е п л ев, Основы расчета воздушных завес, приточных струй и порнстых фнльтров, Стройнздат, 1950.

11. R. F. Davis, The Mechanics of Flame and Air Jets, „Engineering", Vol. 144, No $3750-3752,1937$, p. $608-610$ and $667-668$ 